\title{
Mechanosensing in Bone
}

\author{
Jenneke Klein-Nulend
}

Published online: 26 February 2011

(C) Springer Science+Business Media, LLC 2011

The rationale for this issue of Clinical Reviews in Bone and Mineral Metabolism is rooted in the recognition of the revolution of the role of the osteocyte in bone biology. Since Wolff formulated his law of bone remodeling more than a century ago, the notion that mechanical function determines skeletal design and bone tissue organization has become widely accepted, and the mechanism whereby bone tissue detects mechanical loading is now becoming more and more clear. Basically, the cellular response mechanism to altered load starts with an adequate mechanical signal that is detected by the sensor cells, leading to the generation of an adequate signal that modulates bone formation and/or bone resorption. Bone formation and bone resorption result from the activity of osteoblasts and osteoclasts on the bone surface, while osteocytes are the cells within the tissue proper, and bone lining cells cover inactive bone surfaces. It is now known that osteocytes act as mechanosensors regulating mechanical adaptation and that load placed on an intact bone activates them. Our current knowledge of osteocytes still lags behind what we know of the properties and functions of both osteoblasts and osteoclasts, but the pace with which our knowledge of the osteocyte is advancing is impressive and illustrates an important role for osteocytes in bone biology.

This issue of Clinical Reviews in Bone and Mineral Metabolism on "Mechanosensing in bone" is intended to

\footnotetext{
J. Klein-Nulend $(\square)$

Department of Oral Cell Biology, Academic Centre for Dentistry, Amsterdam-University of Amsterdam and VU University Amsterdam, Research Institute MOVE, Gustav Mahlerlaan 3004, 1081 LA Amsterdam, The Netherlands e-mail: j.kleinnulend@acta.nl
}

review the concept and background information about the topic of mechanosensing in bone with the latest information on putative chemical/mechanical regulators along with speculation/impact on disease manifestations and is designed with a logic that might compel to read on.

The first chapter, "Mechanisms of osteocyte mechanotransduction" by Bakker and Klein-Nulend, covers and provides a critical analysis of our current knowledge on how bone transduction takes place from the level of mechanical loads placed upon the bone as an organ to activation of a cell.

The second chapter, "Application of bioimaging to osteocyte biology" by Kamioka and Adachi, is specifically devoted to the application of a new bioimaging technique to reveal the morphology of osteocytes as well as part of their biological nature. The application of this new technique has important relevance for understanding the osteocytes behavior in response to an extracellular stimulus.

The third chapter, "Stress response by bone cells and implications on microgravity environment" by Bacabac and Van Loon, addresses the great advances that have been made in understanding the mechanisms involved in the adaptation of bone tissue to mechanical loading, leading to the notion that the mechanical properties of cells are a prerequisite to their tissue-level emergent mechanosensing behavior. A condition of disturbed mechanics due to extreme unloading, as in an environment under microgravity, may lead to impaired mechanosensing.

The fourth chapter, "Gap junctions and biophysical regulation of bone cells" by Lloyd and Donahue, demonstrates the integral role of gap junctional intercellular communication in skeletal physiology and bone cell mechanosensing. This direct cell-cell communication via gap junctions is important for the communication between osteoblasts, osteoclasts, and osteocytes and is integral to 
their ability to build and maintain the skeletal system and respond to physical signals.

The fifth chapter, "Primary cilia-mediated mechanotransduction in bone" by Lee, Hoey, and Jacobs, discusses growing evidence supporting primary cilia, single immotile organelles that extend from the surface of bone cells, as mediators of mechanically regulated skeletal homeostasis and development.

The sixth chapter, "The load-bearing mechanosome revisited" by Bidwell and Pavalko, covers the significant progress related to the mechanosome concept since the hypothesis was originally published in 2003. The mechanosome hypothesis is a heuristic model for investigating mechanotransduction in bone, suggesting specific approaches for investigating how mechanical information is conveyed from the membrane of the sensor bone cell to the target genes and how this transmitted information from the membrane is converted into changes in transcription.

The seventh chapter, "Osteocytes in normal physiology and osteoporosis" by Watanabe and Ikeda, focuses on the physiologic function of osteocytes in the regulation of bone and mineral metabolism, summarizing the findings from human disease and mouse genetics, and then extending the discussion to the pathogenetic roles of osteocytes in skeletal aging.

Finally, I am indebted to all the authors for delivering their chapters on time. I trust that the work will be useful to you-enjoy their work! 